\title{
Existence and approximation of a nonlinear degenerate parabolic system modelling acid-mediated tumour invasion
}

\author{
JOHN W. BARRETT \\ Department of Mathematics, Imperial College, London SW7 2AZ, UK \\ E-mail:jwb@ic.ac.uk
}

KLAUS DECKELNICK

Institut für Analysis and Numerik, Otto-von-Guericke-Universität Magdeburg, Universitätsplatz 2, 39106 Magdeburg, Germany

E-mail: klaus.deckelnick@mathematik.uni-magdeburg.de

[Received 21 June 2011 and in revised form 15 June 2012]

\begin{abstract}
We consider a nonlinear parabolic system of reaction-diffusion equations modelling acid-mediated tumour invasion. The system couples potentially degenerate equations for the cell densities of the normal and tumour populations to a parabolic equation for the concentration of $\mathrm{H}^{+}$ions. We obtain an existence result for the system by constructing a suitable finite element approximation and analyzing its convergence. Finally, we report on corresponding numerical experiments.
\end{abstract}

2010 Mathematics Subject Classification: Primary 35K55, 35K65, 65M12, 65M60, 35Q92.

Keywords: Degenerate parabolic system, porous medium equation, existence, finite elements, tumour invasion

\section{Introduction}

Let $\Omega$ be a bounded domain in $\mathbb{R}^{d}, d=1,2$ or 3, with a Lipschitz boundary $\partial \Omega$ having normal $v$. For a fixed positive time $T>0$, we set $\Omega_{T}:=\Omega \times(0, T)$. We consider the following degenerate nonlinear parabolic system.

$\left(\mathbf{P}_{z}\right)$ Find functions $u_{1}, u_{2}, z: \overline{\Omega_{T}} \rightarrow \mathbb{R}_{\geqslant 0}$ such that for $i=1,2$

$$
\begin{aligned}
& \frac{\partial u_{i}}{\partial t}-\nabla \cdot\left(b\left(u_{1}+u_{2}\right) \nabla u_{i}\right)=r_{i} b\left(u_{1}+u_{2}\right) u_{i}-G_{i}(z) u_{i} \quad \text { in } \Omega_{T}, \\
& u_{i}(\cdot, 0)=u_{i}^{0}(\cdot) \quad \text { in } \Omega, \quad b\left(u_{1}+u_{2}\right) \nabla u_{i} \cdot v=0 \quad \text { on } \partial \Omega \times(0, T),
\end{aligned}
$$

and

$$
\begin{array}{ll}
\frac{\partial z}{\partial t}-\alpha \nabla^{2} z=r_{3} u_{2}-\delta_{3}(z-1) & \text { in } \Omega_{T} \\
z(\cdot, 0)=z^{0}(\cdot) \quad \text { in } \Omega, \quad \nabla z \cdot v=0 & \text { on } \partial \Omega \times(0, T) ;
\end{array}
$$

where, for $i=1,2, G_{i} \in C\left(\mathbb{R},\left[0, \delta_{i}\right]\right)$ is defined by

$$
G_{i}(s):=\delta_{i}\left[1-\exp \left\{-\left(\frac{s-z_{i}^{\text {opt }}}{2 z_{i}^{\text {width }}}\right)^{2}\right\}\right] \text { with } \quad z_{i}^{\text {opt }}, z_{i}^{\text {width }} \in \mathbb{R}_{>0}
$$


and $b \in C(\mathbb{R}, \mathbb{R})$ by

$$
b(s):=(1-s)
$$

In addition, we have that

$$
\begin{gathered}
u_{i}^{0}, z^{0} \in C\left(\bar{\Omega}, \mathbb{R}_{\geqslant 0}\right), \quad i=1,2, \quad \text { and } u_{1}^{0}(x)+u_{2}^{0}(x) \leqslant 1, \quad \forall x \in \bar{\Omega} \\
\delta_{i}, r_{i} \in \mathbb{R}_{\geqslant 0}, \quad i=1,2,3, \quad \text { and } \quad \alpha \in \mathbb{R}_{>0} .
\end{gathered}
$$

The above reaction-diffusion system was introduced in Gatenby et al. [5] to model acid mediated tumour invasion. Here $u_{1}, u_{2}$ are the normalized cell densities of the normal and tumour populations, respectively; and $z$ is the normalized concentration of $H^{+}$ions. The (reaction) terms on the right-hand side of (1.1a) and (1.1b) ensure that $u_{1}, u_{2}, z \geqslant 0$ with $u_{1}+u_{2} \leqslant 1$ a.e. in $\Omega_{T}$, and so the diffusion coefficient $b\left(u_{1}+u_{2}\right)$ in (1.1a) possibly degenerates to zero.

In this simple model, it is assumed that only normal and tumour cells compete for the available space, with growth rates dependent on $r_{1}$ and $r_{2}$, respectively, that fall to zero when the space is fully occupied. These cells can also migrate through space via Fickian diffusion with a densitydependent diffusion coefficient, $b(\cdot)$, which takes on its maximum value, normalized to be unity, in the absence of cells and drops to zero when the space is fully occupied. In addition, it is assumed that each type of cell has an optimal $p H$ value for survival, with a higher $\mathrm{H}^{+}$concentration favoring tumour cells. Perturbations from these optimal values in either the acidic or alkaline direction causes death of those cells, with the death rate saturating when the environment is extremely acidic or alkaline. The above effect is modelled by the $G_{i}(z) u_{i}$ terms on the right-hand side of (1.1a). Finally, the normalized $\mathrm{H}^{+}$ion concentration is assumed to grow at a rate $r_{3}$ proportional to the local concentration of tumour cells, but is also effected by buffering and vascular evacuation. Here the normalized background $\mathrm{H}^{+}$concentration is unity. For more details concerning the model, see Gatenby et al. [5]; and for related models, see Gatenby and Gawalinski [4] and Martin et al. [7].

The key mathematical difficulty in proving existence of a solution to this nonlinear degenerate system is that the diffusion coefficient of the $u_{i}$ equation degenerates in terms of $u_{1}+u_{2}$, and not just $u_{i}$. As a consequence, only weak estimates on $u_{1}$ and $u_{2}$ can be obtained which complicates the existence theory while a uniqueness result seems currently to be out of reach.

Let us briefly refer to two other degenerate parabolic systems that have recently been studied. In [1], the present authors have analyzed a doubly degenerate nonlinear parabolic system, where the diffusion coefficient $b\left(u_{i}, v\right)$ is of the form $u_{i}^{q} v^{s}$ for given $q \geqslant 2$ and $s>0$. However, there $v$ satisfied a reaction-diffusion equation coupled to $u_{i}$ only through the reaction term; making it possible to prove $L^{2}\left(0, T ; W^{2, p}(\Omega)\right)$, with $p>\max \{d, 2\}$, regularity for $v$. This in turn made it possible to prove a uniqueness result, and an error bound for a fully practical finite element approximation of that doubly degenerate nonlinear parabolic system. In [2], the authors study a degenerate parabolic system modelling tumour growth with contact inhibition. In that system, the diffusive terms take the form $\nabla \cdot\left(u_{i} \nabla V\left(u_{1}+u_{2}\right)\right), i=1$, 2, where, e.g., $V(s)=s$ and $u_{1}, u_{2}$ are the densities of normal and abnormal cells.

For ease of exposition, we will first consider a reduced version of $\left(\mathrm{P}_{z}\right)$, where the effect of $z$, the normalized concentration of $\mathrm{H}^{+}$ions, is ignored; that is: 
(P) Find functions $u_{1}, u_{2}: \overline{\Omega_{T}} \rightarrow \mathbb{R}_{\geqslant 0}$ such that for $i=1,2$

$$
\begin{aligned}
\frac{\partial u_{i}}{\partial t}-\nabla \cdot\left(b\left(u_{1}+u_{2}\right) \nabla u_{i}\right) & =r_{i} b\left(u_{1}+u_{2}\right) u_{i} & & \text { in } \Omega_{T}, \\
u_{i}(\cdot, 0) & =u_{i}^{0}(\cdot) & & \text { in } \Omega, \\
b\left(u_{1}+u_{2}\right) \nabla u_{i} \cdot v & =0 & & \text { on } \partial \Omega \times(0, T) .
\end{aligned}
$$

The above system retains the major mathematical difficulty, the novel form of the degenerate diffusion coefficient.

DEFINITION 1.1 A pair $\left\{u_{1}, u_{2}\right\}$ is called a weak solution of (P), (1.4a)-(1.4c), if

(a) $u_{i} \in L^{\infty}\left(\Omega_{T}\right)$ with $u_{i} \geqslant 0$ a.e. in $\Omega_{T}, i=1,2$.

(b) $\frac{\partial u_{i}}{\partial t} \in L^{2}\left(0, T ;\left(H^{1}(\Omega)\right)^{\prime}\right), i=1,2$.

(c) $u:=u_{1}+u_{2} \in C\left(\Omega_{T}\right)$ with $u \leqslant 1$ in $\Omega_{T}$.

(d) For $i=1,2, \int_{u}\left|b(u) \nabla u_{i}\right|^{2} \mathrm{~d} x \mathrm{~d} t<\infty$ and $\frac{\partial u_{i}}{\partial x_{j}} \in L_{\mathrm{loc}}^{2}(u), j=1, \ldots, d$, where $u:=$ $\left\{(x, t) \in \Omega_{T}: b(u)(x, t)>0\right\}$.

(e) For $i=1,2$,

$$
\int_{0}^{T}\left\langle\frac{\partial u_{i}}{\partial t}, \zeta\right\rangle_{H^{1}(\Omega)} \mathrm{d} t+\int_{u} b(u) \nabla u_{i} \cdot \nabla \zeta \mathrm{d} x \mathrm{~d} t=r_{i} \int_{\Omega_{T}} b(u) u_{i} \zeta \mathrm{d} x \mathrm{~d} t
$$

for all $\zeta \in L^{2}\left(0, T ; H^{1}(\Omega)\right)$, with $u_{i}(\cdot, 0)=u_{i}^{0}(\cdot)$.

Our aim is to prove the existence of a weak solution to $(\mathrm{P})$ by constructing a finite element approximation and by analyzing the convergence of these approximate solutions.

The outline of this paper is as follows. In the next section we introduce a fully practical finite element approximation of $(\mathrm{P})$, and establish stability bounds. In Section 3 we prove (subsequence) convergence of this approximation, as the spatial and temporal discretization parameters tend to zero, to a weak solution of $(\mathrm{P})$ in the sense of Definition 1.1. In Section 4 we extend the results of Sections 2 and 3 to the full model $\left(\mathrm{P}_{z}\right)$. Finally, in Section 5 we report on some numerical experiments.

We end this section with a few comments about notation. We adopt the standard notation for Sobolev spaces, denoting the norm of $W^{m, q}(G)(m \in \mathbb{N}, q \in[1, \infty]$ and $G$ a bounded domain in $\mathbb{R}^{d}$ with a Lipschitz boundary) by $\|\cdot\|_{m, q, G}$ and the semi-norm by $|\cdot|_{m, q, G}$. For $q=2, W^{m, 2}(G)$ will be denoted by $H^{m}(G)$ with the associated norm and semi-norm written, as respectively, $\|\cdot\|_{m, G}$ and $|\cdot|_{m, G}$. For ease of notation, in the common case when $G \equiv \Omega$ the subscript " $\Omega$ " will be dropped on the above norms and semi-norms. We introduce also $\left(W^{m, q}(\Omega)\right)^{\prime}$, as the dual space of $W^{m, q}(\Omega)$, and denote its norm by $\|\cdot\|_{\left(W^{m, q}(\Omega)\right)^{\prime}}$. These are naturally extended to vector functions. Throughout $(\cdot, \cdot)$ denotes the standard $L^{2}$ inner product over $\Omega$, and $\langle., .\rangle_{W^{m, q}(\Omega)}$ denotes the duality pairing between $\left(W^{m, q}(\Omega)\right)^{\prime}$ and $W^{m, q}(\Omega)$. We denote the characteristic function of a set $G$ by $\chi_{G}$. In addition, $C$ denotes a generic constant independent of the mesh parameters $h, \tau$. 
Finally, we recall the following compactness result, see Simon [8]. Let $y_{0}, y$ and $y_{1}$ be Banach spaces with a compact embedding $y_{0} \hookrightarrow y$ and a continuous embedding $y \hookrightarrow y_{1}$. Then, for $\mu_{i} \geqslant 1, i=0,1$, the following embedding is compact :

$$
\left\{\eta \in L^{\mu_{0}}\left(0, T ; y_{0}\right): \frac{\partial \eta}{\partial t} \in L^{\mu_{1}}\left(0, T ; y_{1}\right)\right\} \hookrightarrow L^{\mu_{0}}(0, T ; y)
$$

\section{Fully discrete approximation}

We consider the finite element approximation of $(\mathrm{P})$ under the following assumptions on the mesh:

(A) Let $\Omega$ be a polyhedral domain and $\left\{T^{h}\right\}_{h>0}$ a quasi-uniform family of partitionings of $\Omega$ into disjoint open simplices $\kappa$ with $h_{\kappa}:=\operatorname{diam}(\kappa)$ and $h:=\max _{\kappa \in T^{h}} h_{\kappa}$, so that $\bar{\Omega}=\cup_{\kappa \in T^{h}} \bar{\kappa}$. In addition, we assume that this partitioning is acute; that is, the angle between any two sides if $d=2$ (faces if $d=3$ ) of any simplex does not exceed $\frac{\pi}{2}$.

Associated with $T^{h}$ is the finite element space

$$
S^{h}:=\left\{\chi \in C(\bar{\Omega}):\left.\chi\right|_{\kappa} \text { is linear } \forall \kappa \in T^{h}\right\} \subset H^{1}(\Omega) .
$$

Let $\left\{p_{j}\right\}_{j=1}^{J}$ be the coordinates of the nodes of $T^{h}$. Let $\left\{\chi_{j}\right\}_{j=1}^{J}$ be the standard basis functions for $S^{h}$; that is, $\chi_{j} \in S^{h}$ and $\chi_{j}\left(p_{i}\right)=\delta_{i j}$ for $i, j=1, \ldots, J$. We introduce $I^{h}: C(\bar{\Omega}) \rightarrow S^{h}$, the interpolation operator, such that $I^{h} \eta\left(p_{j}\right)=\eta\left(p_{j}\right)$ for $j=1, \ldots, J$. A discrete semi-inner product on $C(\bar{\Omega})$ is then defined by

$$
\left(\eta_{1}, \eta_{2}\right)^{h}:=\int_{\Omega} I^{h}\left(\eta_{1}(x) \eta_{2}(x)\right) \mathrm{d} x \equiv \sum_{j=1}^{J} m_{j} \eta_{1}\left(p_{j}\right) \eta_{2}\left(p_{j}\right)
$$

where $m_{j}:=\left(1, \chi_{j}\right)>0$. We define $\|\eta\|_{h}:=\left[(\eta, \eta)^{h}\right]^{\frac{1}{2}}$ for all $\eta \in C(\bar{\Omega})$. Finally, we introduce the $L^{2}$ projections $P^{h}, Q^{h}: L^{2}(\Omega) \rightarrow S^{h}$ defined by

$$
\left(P^{h} \eta, \chi\right)=\left(Q^{h} \eta, \chi\right)^{h}=(\eta, \chi), \quad \forall \chi \in S^{h} .
$$

Let $I$ denote the identity operator. Below we recall some well-known results concerning $S^{h}$ for any $\kappa \in T^{h}, \chi, z^{h} \in S^{h}, m \in\{0,1\}, p \in[1, \infty]:$

$$
\begin{array}{rlrl}
|\chi|_{1, \kappa} & \leqslant C_{1} h_{\kappa}^{-1}|\chi|_{0, \kappa} \leqslant C_{2} h^{-1}|\chi|_{0, \kappa}, & \\
|\chi|_{1, r, \kappa} \leqslant C h_{\kappa}^{-d\left(\frac{1}{p}-\frac{1}{r}\right)-1}|\chi|_{0, p, \kappa}, & \text { for any } r \in[p, \infty], \\
\lim _{h \rightarrow 0}\left|\left(I-I^{h}\right) \eta\right|_{0, \infty} & =0, & \forall \eta \in C(\bar{\Omega}), \\
\left|\left(I-I^{h}\right) \eta\right|_{m} & \leqslant C h^{1-m}|\eta|_{2}, & \\
\int_{\kappa}|\chi|^{2} \mathrm{~d} x & \leqslant \int_{\kappa} I^{h}\left[|\chi|^{2}\right] \mathrm{d} x \leqslant C \int_{\kappa}|\chi|^{2} \mathrm{~d} x, & \\
\left|\left(\chi, z^{h}\right)-\left(\chi, z^{h}\right)^{h}\right| & \leqslant\left|\left(I-I^{h}\right)\left(\chi z^{h}\right)\right|_{0,1} \leqslant C h^{1+m}|\chi|_{m}\left|z^{h}\right|_{1} .
\end{array}
$$


Furthermore, as the mesh is quasi-uniform, we recall the well-known bounds

$$
\begin{aligned}
& \left\|\left(I-P^{h}\right) \eta\right\|_{0} \leqslant C h\|\eta\|_{1}, \quad \forall \eta \in H^{1}(\Omega), \\
& \lim _{h \rightarrow 0}\left\|\left(I-P^{h}\right) \eta\right\|_{1}=0, \quad \forall \eta \in H^{1}(\Omega), \\
& \left\|Q^{h} \eta\right\|_{m} \leqslant C\|\eta\|_{m}, \quad \forall \eta \in H^{m}(\Omega), \quad m=0 \text { or } 1 ;
\end{aligned}
$$

In order to see (2.4c) for $m=1$ we observe that (2.3e), (2.2) and (2.3f) imply

$$
\begin{aligned}
\left\|Q^{h} \eta-P^{h} \eta\right\|_{0}^{2} & \leqslant\left(Q^{h} \eta-P^{h} \eta, Q^{h} \eta-P^{h} \eta\right)^{h} \\
& =\left(P^{h} \eta, Q^{h} \eta-P^{h} \eta\right)-\left(P^{h} \eta, Q^{h} \eta-P^{h} \eta\right)^{h} \leqslant C h\left\|Q^{h} \eta-P^{h} \eta\right\|_{0}\left|P^{h} \eta\right|_{1},
\end{aligned}
$$

so that (2.3a) and the $H^{1}$-stability of $P^{h}$ yield

$$
\left|Q^{h} \eta\right|_{1} \leqslant C_{2} h^{-1}\left\|Q^{h} \eta-P^{h} \eta\right\|_{0}+\left|P^{h} \eta\right|_{1} \leqslant C\left|P^{h} \eta\right|_{1} \leqslant C\|\eta\|_{1} .
$$

In addition, as the mesh is acute, we recall that for both $d=2$ and 3 (cf. [6] for $d=3$ )

$$
\left.\nabla \chi_{i} \cdot \nabla \chi_{j}\right|_{\kappa} \leqslant 0, \quad i \neq j, \quad \forall \kappa \in T^{h} ;
$$

which yields for monotone $g \in C^{0,1}(\mathbb{R})$ with Lipschitz constant $L_{g}$ that

$$
\left|\nabla I^{h}[g(\chi)]\right|^{2} \leqslant L_{g} \nabla \chi \cdot \nabla I^{h}[g(\chi)], \quad \forall \chi \in S^{h}, \quad \forall \kappa \in T^{h} .
$$

We introduce the following explicit finite element approximation of $(\mathrm{P})$ with a uniform time step $\tau>0$ : With $U_{i}^{0}=I^{h} u_{i}^{0}, i=1,2$, for $n=1, \ldots, N$, where $N \tau=T$, find $\left\{U_{1}^{n}, U_{2}^{n}\right\} \in S^{h} \times S^{h}$ such that for $i=1,2$

$$
\left(\frac{U_{i}^{n}-U_{i}^{n-1}}{\tau}, \chi\right)^{h}+\left(b\left(U^{n-1}\right) \nabla U_{i}^{n-1}, \nabla \chi\right)=r_{i}\left(b\left(U^{n-1}\right) U_{i}^{n-1}, \chi\right)^{h}, \quad \forall \chi \in S^{h} .
$$

Here, $U^{n-1}:=U_{1}^{n-1}+U_{2}^{n-1}$. Clearly, as the scheme is explicit, the existence and uniqueness of $\left\{U_{1}^{n}, U_{2}^{n}\right\}$ follows trivially. In order to analyze the scheme we shall assume throughout the paper that the following time step restriction is satisfied:

$$
\tau \leqslant \frac{h^{2}}{C_{2}^{2}+h^{2} \max \left\{r_{1}, r_{2}\right\}},
$$

where $C_{2}$ is as defined in (2.3a). Below we derive a-priori estimates on the solution $\left\{U_{1}^{n}, U_{2}^{n}\right\}$, as well as on $U^{n}$, for $n=0, \ldots, N$.

LEMMA 2.1 We have for $n=0, \ldots, N$

$$
U_{i}^{n}(x) \geqslant 0, \quad i=1,2, \quad \text { and } \quad U^{n}(x) \leqslant 1 \quad \text { for all } x \in \bar{\Omega} .
$$

Proof. The assertion holds for $n=0$ in view of our assumptions on $u_{i}^{0}, i=1,2$. Suppose now that it is true for some $n-1 \in\{0, \ldots, N-1\}$. Fixing $j \in\{1, \ldots, J\}$ and inserting $\chi=\chi_{j}$ into (2.7) 
we obtain that

$$
\begin{aligned}
m_{j} U_{i}^{n}\left(p_{j}\right)= & m_{j} U_{i}^{n-1}\left(p_{j}\right)\left[1+\tau r_{i} b\left(U^{n-1}\left(p_{j}\right)\right)\right] \\
& -\tau \sum_{k=1}^{J} U_{i}^{n-1}\left(p_{k}\right)\left[\left.\sum_{\kappa \in T^{h}}\left(\nabla \chi_{k} \cdot \nabla \chi_{j}\right)\right|_{\kappa} \int_{\kappa} b\left(U^{n-1}\right) \mathrm{d} x\right] .
\end{aligned}
$$

In view of (2.5) and (2.3a), and observing that $b\left(U^{n-1}\right), \chi_{j} \in[0,1]$ we deduce that

$$
\begin{aligned}
m_{j} U_{i}^{n}\left(p_{j}\right) & \geqslant U_{i}^{n-1}\left(p_{j}\right)\left[m_{j}-\tau \sum_{\kappa \in T^{h}} \int_{\kappa}\left|\nabla \chi_{j}\right|^{2} \mathrm{~d} x\right] \\
& \geqslant U_{i}^{n-1}\left(p_{j}\right)\left[m_{j}-C_{2}^{2} \tau h^{-2} \sum_{\kappa \in T^{h}} \int_{\kappa} \chi_{j} \mathrm{~d} x\right] \\
& =m_{j} U_{i}^{n-1}\left(p_{j}\right)\left(1-C_{2}^{2} \tau h^{-2}\right) \geqslant 0,
\end{aligned}
$$

as $C_{2}^{2} \tau h^{-2} \leqslant 1$. Hence, we have proved the first result in (2.9).

On the other hand, (2.10) yields that

$$
\begin{aligned}
m_{j} U^{n}\left(p_{j}\right)=m_{j}\left[U^{n-1}\left(p_{j}\right)\right. & \left.+\tau b\left(U^{n-1}\left(p_{j}\right)\right)\left(r_{1} U_{1}^{n-1}\left(p_{j}\right)+r_{2} U_{2}^{n-1}\left(p_{j}\right)\right)\right] \\
& -\tau \sum_{k=1}^{J} U^{n-1}\left(p_{k}\right)\left[\left.\sum_{\kappa \in T^{h}}\left(\nabla \chi_{k} \cdot \nabla \chi_{j}\right)\right|_{\kappa} \int_{\kappa} b\left(U^{n-1}\right) \mathrm{d} x\right] .
\end{aligned}
$$

Using again (2.5), (2.3a) and $b\left(U^{n-1}\right), \chi_{j} \in[0,1]$, as well as the facts that $U^{n-1} \in[0,1]$ and $\sum_{k=1}^{J} \nabla \chi_{k} \equiv 0$, we obtain that

$$
\begin{aligned}
\left.\sum_{k=1}^{J} U^{n-1}\left(p_{k}\right) \sum_{\kappa \in T^{h}}\left(\nabla \chi_{k} \cdot \nabla \chi_{j}\right)\right|_{\kappa} & \int_{\kappa} b\left(U^{n-1}\right) \mathrm{d} x \\
\geqslant & \left.\sum_{k \neq j} \sum_{\kappa \in T^{h}}\left(\nabla \chi_{k} \cdot \nabla \chi_{j}\right)\right|_{\kappa} \int_{\kappa} b\left(U^{n-1}\right) \mathrm{d} x \\
& \quad+\left.\sum_{\kappa \in T^{h}} U^{n-1}\left(p_{j}\right)\left|\nabla \chi_{j}\right|^{2}\right|_{\kappa} \int_{\kappa} b\left(U^{n-1}\right) \mathrm{d} x \\
\geqslant & \left.\sum_{\kappa \in T^{h}}\left(U^{n-1}\left(p_{j}\right)-1\right)\left|\nabla \chi_{j}\right|^{2}\right|_{\kappa} \int_{\kappa} b\left(U^{n-1}\right) \mathrm{d} x \\
\geqslant & m_{j} C_{2}^{2} h^{-2}\left(U^{n-1}\left(p_{j}\right)-1\right) .
\end{aligned}
$$

If we insert this bound into (2.12) we deduce, on noting that $U^{n-1} \in[0,1]$, that

$$
\begin{aligned}
m_{j} U^{n}\left(p_{j}\right) \leqslant & m_{j}\left[U^{n-1}\left(p_{j}\right)+\tau C_{2}^{2} h^{-2}\left(1-U^{n-1}\left(p_{j}\right)\right)\right] \\
& +m_{j} \tau \max \left\{r_{1}, r_{2}\right\}\left(1-U^{n-1}\left(p_{j}\right)\right) U^{n-1}\left(p_{j}\right) \\
\leqslant & m_{j},
\end{aligned}
$$


as $\tau\left(C_{2}^{2} h^{-2}+\max \left\{r_{1}, r_{2}\right\}\right) \leqslant 1$. Hence, we have proved the second result in (2.9).

Lemma 2.2 The solution $\left\{U_{1}^{n}, U_{2}^{n}\right\}$ of (2.7) satisfies for $i=1,2$

$$
\begin{aligned}
& \max _{n=0, \ldots, N}\left\|U_{i}^{n}\right\|_{0}^{2}+\sum_{n=1}^{N} \tau \int_{\Omega} b\left(U^{n-1}\right)\left|\nabla U_{i}^{n-1}\right|^{2} \mathrm{~d} x \leqslant C, \\
& \sum_{n=1}^{N}\left\|U_{i}^{n}-U_{i}^{n-1}\right\|_{0}^{2}+\sum_{n=1}^{N} \tau\left\|\frac{U_{i}^{n}-U_{i}^{n-1}}{\tau}\right\|_{\left(H^{1}(\Omega)\right)^{\prime}}^{2} \leqslant C .
\end{aligned}
$$

Proof. Choosing $\chi=U_{i}^{n}$ in (2.7) we obtain, on noting (2.9) and that $b\left(U^{n-1}\right) \in[0,1]$, for $i=1,2$ that

$$
\begin{aligned}
& \frac{1}{2}\left[\left\|U_{i}^{n}\right\|_{h}^{2}-\left\|U_{i}^{n-1}\right\|_{h}^{2}+\left\|U_{i}^{n}-U_{i}^{n-1}\right\|_{h}^{2}\right]+\tau \int_{\Omega} b\left(U^{n-1}\right)\left|\nabla U_{i}^{n-1}\right|^{2} \mathrm{~d} x \\
& \quad \leqslant \tau\left(r_{i}|\Omega|+\int_{\Omega} \sqrt{b\left(U^{n-1}\right)}\left|\nabla U_{i}^{n-1}\right|\left|\nabla\left(U_{i}^{n}-U_{i}^{n-1}\right)\right| \mathrm{d} x\right) \\
& \quad \leqslant \frac{3 \tau}{4} \int_{\Omega} b\left(U^{n-1}\right)\left|\nabla U_{i}^{n-1}\right|^{2} \mathrm{~d} x+\frac{\tau}{3} C_{2}^{2} h^{-2}\left\|U_{i}^{n}-U_{i}^{n-1}\right\|_{0}^{2}+C \tau,
\end{aligned}
$$

where we have again applied (2.3a) as well as the elementary inequality $a b \leqslant \frac{3}{4} a^{2}+\frac{1}{3} b^{2}$. Summing the above from $n=1$ to $m$, for $m=1, \ldots, N$, and taking into account $(2.3 \mathrm{e})$ and the fact that $C_{2}^{2} \tau h^{-2} \leqslant 1$ yields (2.14a) and the first bound in (2.14b).

In order to prove the second bound in $(2.14 \mathrm{~b})$, we take an arbitrary $\zeta \in H^{1}(\Omega)$. Choosing $\chi=Q^{h} \zeta$ in (2.7) and noting (2.2) we obtain for $i=1,2$ that

$$
\begin{aligned}
\left(\frac{U_{i}^{n}-U_{i}^{n-1}}{\tau}, \zeta\right) & =\left(\frac{U_{i}^{n}-U_{i}^{n-1}}{\tau}, Q^{h} \zeta\right)^{h} \\
& =-\int_{\Omega} b\left(U^{n-1}\right) \nabla U_{i}^{n-1} \cdot \nabla Q^{h} \zeta \mathrm{d} x+r_{i}\left(b\left(U^{n-1}\right) U_{i}^{n-1}, Q^{h} \zeta\right)^{h} .
\end{aligned}
$$

Using the stability properties of $Q^{h},(2.4 \mathrm{c}),(2.9),(2.3 \mathrm{e})$ and that $b\left(U^{n-1}\right) \in[0,1]$, we infer for $i=1,2$ that

$$
\left|\left(\frac{U_{i}^{n}-U_{i}^{n-1}}{\tau}, \zeta\right)\right| \leqslant C\left(\int_{\Omega} b\left(U^{n-1}\right)\left|\nabla U_{i}^{n-1}\right|^{2} \mathrm{~d} x\right)^{\frac{1}{2}}\|\zeta\|_{1}+C\|\zeta\|_{0}
$$

Repeating the above for all $\zeta \in H^{1}(\Omega)$ yields for $i=1,2$ that

$$
\left\|\frac{U_{i}^{n}-U_{i}^{n-1}}{\tau}\right\|_{\left(H^{1}(\Omega)\right)^{\prime}} \leqslant C\left(1+\int_{\Omega} b\left(U^{n-1}\right)\left|\nabla U_{i}^{n-1}\right|^{2} \mathrm{~d} x\right)^{\frac{1}{2}} .
$$

The second bound in (2.14b) now follows from (2.18) and (2.14a).

REMARK 2.1 In order to avoid the restrictive assumption (2.8) one could consider a semi-implicit scheme; that is, (2.7) with $\nabla U_{i}^{n-1}$ replaced by $\nabla U_{i}^{n}$, which requires a linear solve for $U_{i}^{n}, i=1,2$, 
at each time level. It is a simple matter to show that such a scheme satisfies (2.9), (2.14a), with $\nabla U_{i}^{n-1}$ replaced by $\nabla U_{i}^{n}$, and (2.14b) under the mild stability restriction $\tau \max \left\{r_{1}, r_{2}\right\} \leqslant 1$.

However, the convergence analysis in the next section exploits similarities of $\left(\mathrm{P}_{z}\right)$ with the nonhomogeneous porous medium equation. For this, it is crucial that $b(\cdot)$ and the gradient term on the left-hand side of (2.7) are at the same time level; which is true for the explicit scheme, but not for the semi-implicit scheme. Hence, we will restrict ourselves to the explicit scheme in this paper.

\section{Convergence}

Let us define for $i=1,2$ and $n=1, \ldots, N$

$$
\begin{array}{lll}
U_{i ; h, \tau}(x, t):=\frac{t-t_{n-1}}{\tau} U_{i}^{n}(x)+\frac{t_{n}-t}{\tau} U_{i}^{n-1}(x), & t_{n-1} \leqslant t \leqslant t_{n}, \\
U_{i ; h, \tau}^{-}(x, t):=U_{i}^{n-1}(x), & U_{i ; h, \tau}^{+}(x, t):=U_{i}^{n}(x), & t_{n-1}<t \leqslant t_{n},
\end{array}
$$

and $U_{h, \tau}:=U_{1 ; h, \tau}+U_{2 ; h, \tau}, U_{h, \tau}^{ \pm}:=U_{1 ; h, \tau}^{ \pm}+U_{2 ; h, \tau}^{ \pm}$.

We have the following convergence results.

Lemma 3.1 There exists a sequence $\{h, \tau\} \rightarrow\{0,0\}$ satisfying (2.8) such that for $i=1,2$

$$
\begin{array}{rlll}
U_{i ; h, \tau}, U_{i ; h, \tau}^{-} & \stackrel{*}{\rightarrow} u_{i} & \text { in } L^{\infty}\left(\Omega_{T}\right), \\
U_{i ; h, \tau}-U_{i ; h, \tau}^{-} & \rightarrow & 0 & \text { in } L^{2}\left(\Omega_{T}\right), \\
\frac{\partial U_{i ; h, \tau}}{\partial t} & \rightarrow & \frac{\partial u_{i}}{\partial t} & \text { in } L^{2}\left(0, T ;\left(H^{1}(\Omega)\right)^{\prime}\right), \\
b\left(U_{h, \tau}^{-}\right) \nabla U_{i ; h, \tau}^{-} & \rightarrow & F_{i} & \text { in } L^{2}\left(0, T ;\left[L^{2}(\Omega)\right]^{d}\right) ;
\end{array}
$$

where $u_{i}(x, t) \geqslant 0$ and $u(x, t):=u_{1}(x, t)+u_{2}(x, t) \in[0,1]$ for a.e. $(x, t) \in \Omega_{T}$, and $F_{i}$ needs to be identified in terms of $u_{i}$ and $u$, see Lemma 3.5 below.

Proof. The desired results follow immediately from Lemma 2.1 and Lemma 2.2.

Lemma 3.2 There exists a subsequence $\{h, \tau\} \rightarrow\{0,0\}$ of the sequence in (3.2a)-(3.2d) such that

$$
\begin{aligned}
U_{h, \tau}, U_{h, \tau}^{-} & \rightarrow u & \text { a.e. in } \Omega_{T}, \\
{\left[b\left(U_{h, \tau}^{-}\right)\right]^{3} U_{i ; h, \tau}^{-} } & \rightarrow[b(u)]^{3} u_{i} & \text { a.e. in } \Omega_{T}, \quad i=1,2 .
\end{aligned}
$$

Proof. We define $B(s):=[b(s)]^{3}=(1-s)^{3}$ and consider $V_{h, \tau}:=B\left(U_{h, \tau}\right)$. Clearly, $\nabla V_{h, \tau}=$ $B^{\prime}\left(U_{h, \tau}\right) \nabla U_{h, \tau}$ a.e. in $\Omega_{T}$. On $\Omega \times\left[t_{n-1}, t_{n}\right]$, we have in view of (3.1a) and (2.9) that

$$
\left|B^{\prime}\left(U_{h, \tau}\right)\right| \leqslant\left|B^{\prime}\left(U^{n-1}\right)\right|+6\left|U^{n}-U^{n-1}\right| \leqslant 3 \sqrt{b\left(U^{n-1}\right)}+6\left|U^{n}-U^{n-1}\right|,
$$

as well as

$$
\left|\nabla U_{h, \tau}\right| \leqslant\left|\nabla U^{n-1}\right|+\left|\nabla\left(U^{n}-U^{n-1}\right)\right| .
$$


This implies, on noting (2.3a) and (2.3b) with $r=p=\infty$, that

$$
\begin{aligned}
& \int_{0}^{T}\left\|\nabla V_{h, \tau}\right\|_{0}^{2} \mathrm{~d} t=\sum_{n=1}^{N} \int_{t_{n-1}}^{t_{n}} \int_{\Omega}\left|B^{\prime}\left(U_{h, \tau}\right)\right|^{2}\left|\nabla U_{h, \tau}\right|^{2} \mathrm{~d} x \mathrm{~d} t \\
& \leqslant C \sum_{n=1}^{N} \tau \int_{\Omega}\left[b\left(U^{n-1}\right)+\left|U^{n}-U^{n-1}\right|^{2}\right]\left[\left|\nabla U^{n-1}\right|^{2}+\left|\nabla\left(U^{n}-U^{n-1}\right)\right|^{2}\right] \mathrm{d} x \\
& \leqslant C \sum_{n=1}^{N} \tau\left[\int_{\Omega} b\left(U^{n-1}\right)\left|\nabla U^{n-1}\right|^{2} \mathrm{~d} x+\left\|\nabla\left(U^{n}-U^{n-1}\right)\right\|_{0}^{2}\right. \\
& \left.\quad+\left\|\nabla U^{n-1}\right\|_{0, \infty}^{2}\left\|U^{n}-U^{n-1}\right\|_{0}^{2}\right] \\
& \leqslant C \sum_{n=1}^{N} \tau\left[\int_{\Omega} b\left(U^{n-1}\right)\left|\nabla U^{n-1}\right|^{2} \mathrm{~d} x+h^{-2}\left\|U^{n}-U^{n-1}\right\|_{0}^{2}\right] \\
& \leqslant C,
\end{aligned}
$$

where the last inequality follows from (2.14a), (2.14b) and (2.8). We remark that $B(s):=[b(s)]^{2}=$ $(1-s)^{2}$ would be adequate for the argument above. The cubic choice of $B$ is required for the argument below.

Next, we note that

$$
\frac{\partial V_{h, \tau}}{\partial t}=B^{\prime}\left(U_{h, \tau}\right) \frac{\partial U_{h, \tau}}{\partial t}=B^{\prime}\left(U_{h, \tau}\right) \frac{U^{n}-U^{n-1}}{\tau} \quad \text { on } \Omega \times\left[t_{n-1}, t_{n}\right] .
$$

For $p>d$, let $\phi \in W^{1, p}(\Omega) \subset L^{\infty}(\Omega)$. It follows from (2.2) and (2.7) that for $t \in\left[t_{n-1}, t_{n}\right]$

$$
\begin{aligned}
\left\langle\frac{\partial V_{h, \tau}}{\partial t}, \phi\right\rangle_{W^{1, p}(\Omega)} & =\left(\frac{U^{n}-U^{n-1}}{\tau}, B^{\prime}\left(U_{h, \tau}\right) \phi\right)=\left(\frac{U^{n}-U^{n-1}}{\tau}, Q^{h}\left(B^{\prime}\left(U_{h, \tau}\right) \phi\right)\right)^{h} \\
& =-\int_{\Omega} b\left(U^{n-1}\right) \nabla U^{n-1} \cdot \nabla Q^{h}\left(B^{\prime}\left(U_{h, \tau}\right) \phi\right) \mathrm{d} x \\
& \quad+\left(b\left(U^{n-1}\right)\left(r_{1} U_{1}^{n-1}+r_{2} U_{2}^{n-1}\right), Q^{h}\left(B^{\prime}\left(U_{h, \tau}\right) \phi\right)\right)^{h} .
\end{aligned}
$$

Hence, on noting (2.9), that $b\left(U^{n-1}\right) \in[0,1],(2.3 \mathrm{e})$ and (2.4c), we have for $t \in\left[t_{n-1}, t_{n}\right]$ that

$$
\begin{aligned}
& \left|\left\langle\frac{\partial V_{h, \tau}}{\partial t}, \phi\right\rangle_{W^{1, p}(\Omega)}\right| \\
& \quad \leqslant\left(\int_{\Omega} b\left(U^{n-1}\right)\left|\nabla U^{n-1}\right|^{2} \mathrm{~d} x\right)^{\frac{1}{2}}\left\|\nabla Q^{h}\left(B^{\prime}\left(U_{h, \tau}\right) \phi\right)\right\|_{0}+C\left\|Q^{h}\left(B^{\prime}\left(U_{h, \tau}\right) \phi\right)\right\|_{0} \\
& \quad \leqslant C\left(\int_{\Omega} b\left(U^{n-1}\right)\left|\nabla U^{n-1}\right|^{2} \mathrm{~d} x\right)^{\frac{1}{2}}\left\|B^{\prime}\left(U_{h, \tau}\right) \phi\right\|_{1}+C\left\|B^{\prime}\left(U_{h, \tau}\right) \phi\right\|_{0} \\
& \quad \leqslant C\left(\int_{\Omega} b\left(U^{n-1}\right)\left|\nabla U^{n-1}\right|^{2} \mathrm{~d} x\right)^{\frac{1}{2}}\left(\left\|b\left(U_{h, \tau}\right) \nabla U_{h, \tau}\right\|_{0}\|\phi\|_{0, \infty}+\|\phi\|_{1}\right)+C\|\phi\|_{0} .
\end{aligned}
$$

Arguing similarly as in (3.4), it follows for $t \in\left[t_{n-1}, t_{n}\right]$ that

$$
\left\|b\left(U_{h, \tau}\right) \nabla U_{h, \tau}\right\|_{0} \leqslant\left(\int_{\Omega} b\left(U^{n-1}\right)\left|\nabla U^{n-1}\right|^{2} \mathrm{~d} x\right)^{\frac{1}{2}}+C h^{-1}\left\|U^{n}-U^{n-1}\right\|_{0} .
$$


Inserting this bound into (3.5) we deduce for $t \in\left[t_{n-1}, t_{n}\right]$ that

$$
\left|\left\langle\frac{\partial V_{h, \tau}}{\partial t}, \phi\right\rangle_{W^{1, p}(\Omega)}\right| \leqslant C\|\phi\|_{1, p}\left(1+\int_{\Omega} b\left(U^{n-1}\right)\left|\nabla U^{n-1}\right|^{2} \mathrm{~d} x+h^{-2}\left\|U^{n}-U^{n-1}\right\|_{0}^{2}\right) .
$$

Hence (2.14a), (2.14b) and (2.8) imply that

$$
\begin{aligned}
& \int_{0}^{T}\left\|\frac{\partial V_{h, \tau}}{\partial t}\right\|_{\left(W^{1, p}(\Omega)\right)^{\prime}} \mathrm{d} t \\
& \quad \leqslant C\left(1+\sum_{n=1}^{N} \tau \int_{\Omega} b\left(U^{n-1}\right)\left|\nabla U^{n-1}\right|^{2} \mathrm{~d} x+\sum_{n=1}^{N} \tau h^{-2}\left\|U^{n}-U^{n-1}\right\|_{0}^{2}\right) \leqslant C
\end{aligned}
$$

We infer from (3.4), (3.6) and (1.6) that there exists a subsequence $\{h, \tau\} \rightarrow\{0,0\}$, subject to (2.8), such that $\left\{V_{h, \tau}\right\}_{h, \tau}$ converges in $L^{2}\left(\Omega_{T}\right)$ and almost everywhere. Since $U_{h, \tau}=1-V_{h, \tau}^{\frac{1}{3}}$, we see that there exists a subsequence of $\left\{U_{h, \tau}\right\}_{h, \tau}$, on recalling (3.2a), that converges to $u=u_{1}+u_{2}$ almost everywhere; and, on recalling (3.2b), the same is true for $\left\{U_{h, \tau}^{-}\right\}_{h, \tau}$ after possibly extracting a further subsequence. Hence the desired result (3.3a).

To prove (3.3b), we consider $W_{i ; h, \tau}:=B\left(U_{h, \tau}\right) U_{i ; h, \tau}=\left[b\left(U_{h, \tau}\right)\right]^{3} U_{i ; h, \tau} \in L^{\infty}\left(\Omega_{T}\right), i=$ 1,2 , and proceed in the same way as above to show, similarly to (3.4) and (3.6), that

$$
\int_{0}^{T}\left[\left\|\nabla W_{i ; h, \tau}\right\|_{0}^{2}+\left\|\frac{\partial W_{i ; h, \tau}}{\partial t}\right\|_{\left(W^{1, p}(\Omega)\right)^{\prime}}\right] \mathrm{d} t \leqslant C, \quad i=1,2 .
$$

Hence, there exists a further subsequence of $\{h, \tau\} \rightarrow\{0,0\}$, satisfying (2.8), and functions $w_{i} \in$ $L^{\infty}\left(\Omega_{T}\right), i=1,2$, such that

$$
W_{i ; h, \tau} \rightarrow w_{i} \quad \text { a.e. in } \Omega_{T}, \quad i=1,2 .
$$

On noting (3.2a), (3.2b) and (3.3a), it is straightforward to deduce that $w_{i}=[b(u)]^{3} u_{i}, i=1,2$; and that the desired result $(3.3 \mathrm{~b})$ holds.

Our next aim is to establish the regularity of $u$. For this purpose we will first show that $\widetilde{u}:=$ $b(u)=1-u$ is a weak solution of the nonhomogeneous porous medium equation, see, e.g., Vázquez [9],

$$
\begin{gathered}
\frac{\partial \widetilde{u}}{\partial t}-\nabla \cdot(\nabla \psi(\widetilde{u}))=f \quad \text { in } \Omega_{T}, \\
\widetilde{u}(\cdot, 0)=1-u^{0}(\cdot) \quad \text { in } \Omega, \quad \nabla \psi(\widetilde{u}) \cdot v=0 \quad \text { on } \partial \Omega \times(0, T) ;
\end{gathered}
$$

where

$$
\begin{aligned}
& \psi(s):= \begin{cases}\frac{1}{2} \operatorname{sgn}(s) s^{2} & \text { if }|s| \leqslant 1, \\
\operatorname{sgn}(s)\left(|s|-\frac{1}{2}\right) & \text { if }|s|>1 ;\end{cases} \\
& f:=-b(u)\left(r_{1} u_{1}+r_{2} u_{2}\right) \in L^{\infty}\left(\Omega_{T}\right) \quad \text { and } \quad u^{0}:=u_{1}^{0}+u_{2}^{0} .
\end{aligned}
$$

Here for the pursuing analysis, we have extended the domain of $\psi$ from [0,1], which is the range of $\widetilde{u}$, to $\mathbb{R}$ so that $\psi \in C^{1}(\mathbb{R})$ is a strictly increasing odd function. 
LEMmA 3.3 It follows that $\widetilde{u}=1-u \in L^{\infty}\left(\Omega_{T}\right)$ with $\psi(\widetilde{u}) \in L^{2}\left(0, T ; H^{1}(\Omega)\right)$ is such that

$$
\begin{aligned}
-\int_{0}^{T}\left(\widetilde{u}, \frac{\partial \zeta}{\partial t}\right) \mathrm{d} t-\left(1-u_{0}(\cdot), \zeta(\cdot, 0)\right)+\int_{0}^{T}(\nabla \psi(\widetilde{u}), \nabla \zeta) \mathrm{d} t=\int_{0}^{T}(f, \zeta) \mathrm{d} t, \\
\forall \zeta \in L^{2}\left(0, T ; H^{1}(\Omega)\right) \cap H^{1}\left(0, T ; L^{2}(\Omega)\right) \text { with } \zeta(\cdot, T)=0 \text { a.e. in } \Omega .
\end{aligned}
$$

Hence, $\widetilde{u}$ is the unique weak solution of the nonhomogeneous porous medium equation (3.7a)-(3.7c) for the stated data.

Proof. It follows from (2.7) that the function $\widetilde{U}_{h, \tau}:=1-U_{h, \tau}$ satisfies $\widetilde{U}_{h, \tau}(\cdot, 0)=1-I^{h} u^{0}(\cdot)$ and

$$
\begin{aligned}
& \int_{0}^{T}\left[\left(\frac{\partial \widetilde{U}_{h, \tau}}{\partial t}, \chi\right)^{h}+\left(\nabla \psi\left(\widetilde{U}_{h, \tau}^{-}\right), \nabla \chi\right)\right] \mathrm{d} t \\
&=-\int_{0}^{T}\left(b\left(U_{h, \tau}^{-}\right)\left(r_{1} U_{1 ; h, \tau}^{-}+r_{2} U_{2 ; h, \tau}^{-}\right), \chi\right)^{h} \mathrm{~d} t, \quad \forall \chi \in L^{1}\left(0, T ; S^{h}\right) .
\end{aligned}
$$

Let $\zeta \in C^{\infty}\left(\overline{\Omega_{T}}\right)$ with $\zeta(\cdot, T)=0$. Using integration by parts we derive from (3.9) with $\chi=P^{h} \zeta$ that

$$
\begin{array}{r}
-\int_{0}^{T}\left(\widetilde{U}_{h, \tau}, P^{h} \frac{\partial \zeta}{\partial t}\right)^{h} \mathrm{~d} t-\left(\widetilde{U}_{h, \tau}(\cdot, 0), P^{h} \zeta(\cdot, 0)\right)^{h}+\int_{0}^{T}\left(\nabla \psi\left(\widetilde{U}_{h, \tau}^{-}\right), \nabla P^{h} \zeta\right) \mathrm{d} t \\
=-\int_{0}^{T}\left(b\left(U_{h, \tau}^{-}\right)\left(r_{1} U_{1 ; h, \tau}^{-}+r_{2} U_{2 ; h, \tau}^{-}\right), P^{h} \zeta\right)^{h} \mathrm{~d} t
\end{array}
$$

As $\nabla \psi\left(\widetilde{U}_{h, \tau}^{-}\right)=-b\left(U_{h, \tau}^{-}\right) \nabla U_{h, \tau}^{-}$, it follows from (3.2d) and (3.3a) that

$$
\nabla \psi\left(\widetilde{U}_{h, \tau}^{-}\right) \rightarrow-\left(F_{1}+F_{2}\right)=\nabla \psi(\widetilde{u}) \quad \text { in } \quad L^{2}\left(0, T ;\left[L^{2}(\Omega)\right]^{d}\right)
$$

for a subsequence of $\{h, \tau\} \rightarrow\{0,0\}$ satisfying (2.8). We stress here that one cannot apply the chain rule directly to obtain (3.11), as $\nabla \widetilde{u}$ only exists in a distributional sense. We infer from (2.4a) and (2.4b) that $\nabla P^{h} \zeta \rightarrow \nabla \zeta$ in $L^{2}\left(0, T ;\left[L^{2}(\Omega)\right]^{d}\right)$ which together with (3.11) implies that

$$
\int_{0}^{T}\left(\nabla \psi\left(\widetilde{U}_{h, \tau}^{-}\right), \nabla P^{h} \zeta\right) \mathrm{d} t \rightarrow \int_{0}^{T}(\nabla \psi(\widetilde{u}), \nabla \zeta) \mathrm{d} t .
$$

Furthermore, (3.3a), (2.9) and (2.4a) show that $b\left(U_{h, \tau}^{-}\right) P^{h} \zeta \rightarrow b(u) \zeta$ in $L^{1}\left(\Omega_{T}\right)$, so that we deduce with the help of (3.2a), (2.3f) and (2.4b) that

$$
\int_{0}^{T}\left(b\left(U_{h, \tau}^{-}\right)\left(r_{1} U_{1 ; h, \tau}^{-}+r_{2} U_{2 ; h, \tau}^{-}\right), P^{h} \zeta\right)^{h} \mathrm{~d} t \rightarrow \int_{0}^{T}\left(b(u)\left(r_{1} u_{1}+r_{2} u_{2}\right), \zeta\right) \mathrm{d} t .
$$

Treating the remaining terms in a similar way we deduce from (3.10) after passing to the limit $\{h, \tau\} \rightarrow\{0,0\}$

$$
\begin{aligned}
&-\int_{0}^{T}\left(\widetilde{u}, \frac{\partial \zeta}{\partial t}\right) \mathrm{d} t-\left(1-u_{0}(\cdot), \zeta(\cdot, 0)\right)+\int_{0}^{T}(\nabla \psi(\widetilde{u}), \nabla \zeta) \mathrm{d} t \\
&=-\int_{0}^{T}\left(b(u)\left(r_{1} u_{1}+r_{2} u_{2}\right), \zeta\right) \mathrm{d} t, \quad \forall \zeta \in C^{\infty}\left(\overline{\Omega_{T}}\right) \text { with } \zeta(\cdot, T)=0 .
\end{aligned}
$$


Hence, the desired result (3.8) follows on noting a density result for the test functions $\zeta$.

Uniqueness follows in the standard way. If there were two solutions $\widetilde{u}_{i}, i=1,2$, solving (3.8), then subtracting and choosing $\zeta(\cdot, t)=\int_{t}^{T}\left[\psi\left(\widetilde{u}_{1}\right)-\psi\left(\widetilde{u}_{2}\right)\right](\cdot, s) \mathrm{d} s$ yields that

$$
\int_{0}^{T}\left(\widetilde{u}_{1}-\widetilde{u}_{2}, \psi\left(\widetilde{u}_{1}\right)-\psi\left(\widetilde{u}_{2}\right)\right) \mathrm{d} t+\frac{1}{2} \int_{\Omega}\left|\nabla\left(\int_{0}^{T}\left[\psi\left(\widetilde{u}_{1}\right)-\psi\left(\widetilde{u}_{2}\right)\right] \mathrm{d} t\right)\right|^{2} \mathrm{~d} x=0 .
$$

As $\psi$ is strictly increasing, we have that $\widetilde{u}_{1}=\widetilde{u}_{2}$ a.e. in $\Omega_{T}$; and hence the solution of (3.8) is unique.

LEMMA 3.4 The unique weak solution of (3.7a)-(3.7c), $\widetilde{u}$ satisfying (3.8), is such that $\widetilde{u} \in C\left(\Omega_{T}\right)$. Hence, we have that $u \in C\left(\Omega_{T}\right)$ and, in particular, the set $U:=\left\{(x, t) \in \Omega_{T}: b(u)(x, t)>0\right\}$ is open.

Proof. To prove that $\widetilde{u} \in C\left(\Omega_{T}\right)$, we will appeal to Theorem 1 in DiBenedetto [3]. In order to do so, we have to recast our weak form, (3.8), of (3.7a)-(3.7c) into the structure of that theorem.

First, we need a change of dependent variable. Let $\widetilde{w}=\psi(\widetilde{u})$ and so $\widetilde{u}=\beta(\widetilde{w})$, where

$$
\beta(r):= \begin{cases}\operatorname{sgn}(r)(2|r|)^{\frac{1}{2}} & \text { if }|r| \leqslant \frac{1}{2} \\ \operatorname{sgn}(r)\left(|r|+\frac{1}{2}\right) & \text { if }|r|>\frac{1}{2}\end{cases}
$$

It follows that $\beta \in C(\mathbb{R})$ is a strictly increasing function with $\beta(0)=0$ such that (i) $1 \leqslant \beta^{\prime}(r)$ for all $r \in \mathbb{R} \backslash\{0\}$; (ii) $\liminf _{|r| \rightarrow 0} \beta^{\prime}(r)=\infty$; (iii) $\beta^{\prime}(s) \leqslant \beta^{\prime}(r)$ for all $s \in \mathbb{R} \backslash\left[-\frac{1}{2}, \frac{1}{2}\right]$, and for all $r \in\left[-\frac{1}{2}, \frac{1}{2}\right] \backslash\{0\}$; and $\beta^{\prime}$ is decreasing over $\left(0, \frac{1}{2}\right]$ and increasing over $\left[-\frac{1}{2}, 0\right)$. Hence, assumption $\left[\mathrm{A}_{1}\right]$ on $\beta$ in [3] holds. With this change of variables, it follows from (3.8) that $\widetilde{w} \in$ $L^{\infty}\left(\Omega_{T}\right) \cap L^{2}\left(0, T ; H^{1}(\Omega)\right)$ is the unique solution of

$$
\begin{gathered}
-\int_{0}^{T}\left(\beta(\widetilde{w}), \frac{\partial \zeta}{\partial t}\right) \mathrm{d} t-\left(1-u_{0}(\cdot), \zeta(\cdot, 0)\right)+\int_{0}^{T}(\nabla \widetilde{w}, \nabla \zeta) \mathrm{d} t=\int_{0}^{T}(f, \zeta) \mathrm{d} t \\
\forall \zeta \in L^{2}\left(0, T ; H^{1}(\Omega)\right) \cap H^{1}\left(0, T ; L^{2}(\Omega)\right) \text { with } \zeta(\cdot, T)=0 \text { a.e. in } \Omega .
\end{gathered}
$$

In addition, the assumptions $\left[\mathrm{A}_{2}\right]$ and $\left[\mathrm{A}_{3}\right]$ in $[3]$ on the leading order spatial operator and the lower order terms trivially hold.

Finally, we need to check assumption $\left[\mathrm{A}_{4}\right]$ in [3]. For this, we introduce a sequence of $C^{\infty}(\mathbb{R})$ approximations to $\beta$. This is achieved via mollification. Let $\varphi \in C^{\infty}(\mathbb{R})$ be such that

$$
\varphi(r)= \begin{cases}C \exp \left(\left(r^{2}-1\right)^{-1}\right) & \text { if }|r|<1 \\ 0 & \text { if }|r| \geqslant 1\end{cases}
$$

where $C$ is chosen so that $\int_{-\infty}^{\infty} \varphi(r) \mathrm{d} r=1$. Next we introduce for $m \in \mathbb{N}$

$$
\varphi_{m}(r)=m \varphi(m r) \quad \text { for } r \in \mathbb{R}
$$

satisfying $\varphi_{m} \in C^{\infty}(\mathbb{R})$ with $\operatorname{supp}\left(\varphi_{m}\right) \subset\left[-\frac{1}{m}, \frac{1}{m}\right]$ and $\int_{-\infty}^{\infty} \varphi_{m}(r) \mathrm{d} r=1$. For $m \in \mathbb{N}$, we then set

$$
\beta_{m}(r)=\int_{0}^{r} \beta_{m}^{\prime}(s) \mathrm{d} s \quad \text { for } \quad r \in \mathbb{R}
$$


where for $r \in \mathbb{R}$

$$
\beta_{m}^{\prime}(r):=\int_{0}^{\infty} \varphi_{m}(r-s) \beta^{\prime}\left(s+\frac{1}{m}\right) \mathrm{d} s+\int_{-\infty}^{0} \varphi_{m}(r-s) \beta^{\prime}\left(s-\frac{1}{m}\right) \mathrm{d} s .
$$

It follows that $\beta_{m} \in C^{\infty}(\mathbb{R})$, with $\beta_{m}(0)=0$, is a strictly increasing odd function for $m \in \mathbb{N}$. Moreover, we have for $m \in \mathbb{N}$ that

$$
1 \leqslant \beta_{m}^{\prime}(r) \leqslant \beta^{\prime}(r) \quad \forall r \in \mathbb{R} \backslash\{0\} ;
$$

and hence (2.2) in [3] holds. Let $r>0$ be fixed, then for $m$ sufficiently large we have from (3.18), (3.19), (3.16) and (3.15) that

$$
\begin{aligned}
0 \leqslant \beta^{\prime}(r)-\beta_{m}^{\prime}(r) & =\int_{0}^{\infty} \varphi_{m}(r-s)\left[\beta^{\prime}(r)-\beta^{\prime}\left(s+\frac{1}{m}\right)\right] \mathrm{d} s \\
& =m \int_{r-\frac{1}{m}}^{r+\frac{1}{m}} \varphi(r-s)\left[\beta^{\prime}(r)-\beta^{\prime}\left(s+\frac{1}{m}\right)\right] \mathrm{d} s \\
& \leqslant C m \int_{r-\frac{1}{m}}^{r+\frac{1}{m}}\left[\beta^{\prime}(r)-\beta^{\prime}\left(s+\frac{1}{m}\right)\right] \mathrm{d} s .
\end{aligned}
$$

On noting that $\beta^{\prime}$ is uniformly continuous on compact subsets of $\mathbb{R} \backslash\{0\}$, we deduce from (3.20), and on applying a similar argument to (3.20) for a point $r<0$, that

$$
\lim _{m \rightarrow \infty}\left|\beta^{\prime}(r)-\beta_{m}^{\prime}(r)\right|=0 \quad \text { for a.e. } r \in \mathbb{R} .
$$

As $\beta^{\prime}$ is uniformly continuous on compact subsets of $\mathbb{R} \backslash\{0\}$, the argument above is easily adapted to show that

$$
\beta_{m}^{\prime} \rightarrow \beta^{\prime} \quad \text { uniformly on compact subsets of } \mathbb{R} \backslash\{0\} \text { as } m \rightarrow \infty \text {. }
$$

It follows from (3.17), (3.22), (3.21) and Lebesgue's dominated convergence theorem that

$$
\beta_{m} \rightarrow \beta \quad \text { uniformly on compact subsets of } \mathbb{R} \text { as } m \rightarrow \infty \text {. }
$$

Hence, the first part of assumption $\left[\mathrm{A}_{4}\right]$ in [3] holds.

For $m \in \mathbb{N}$, let $\widetilde{w}_{m}$ be the unique solution of the regularized version of (3.14); that is,

$$
\begin{aligned}
& -\int_{0}^{T}\left(\beta_{m}\left(\widetilde{w}_{m}\right), \frac{\partial \zeta}{\partial t}\right) \mathrm{d} t-\left(1-u_{0}(\cdot), \zeta(\cdot, 0)\right)+\int_{0}^{T}\left(\nabla \widetilde{w}_{m}, \nabla \zeta\right) \mathrm{d} t=\int_{0}^{T}(f, \zeta) \mathrm{d} t, \\
& \forall \zeta \in L^{2}\left(0, T ; H^{1}(\Omega)\right) \cap H^{1}\left(0, T ; L^{2}(\Omega)\right) \text { with } \zeta(\cdot, T)=0 \text { a.e. in } \Omega .
\end{aligned}
$$

As $\beta_{m} \in C^{\infty}(\mathbb{R})$ is neither singular nor degenerate, recall (3.19), then standard parabolic theory yields that $\widetilde{u}_{m} \equiv \beta_{m}\left(\widetilde{w}_{m}\right) \in L^{2}\left(0, T ; H^{1}(\Omega)\right) \cap H^{1}\left(0, T ;\left(H^{1}(\Omega)\right)^{\prime}\right)$, with $\widetilde{u}_{m}(\cdot, 0)=1-u^{0}(\cdot)$, is the unique solution of

$$
\begin{aligned}
\int_{0}^{T}\left\langle\frac{\partial \widetilde{u}_{m}}{\partial t}, \zeta\right\rangle_{H^{1}(\Omega)} \mathrm{d} t+\int_{0}^{T}\left(\nabla \psi_{m}\left(\widetilde{u}_{m}\right), \nabla \zeta\right) \mathrm{d} t= & \int_{0}^{T}(f, \zeta) \mathrm{d} t, \\
& \forall \zeta \in L^{2}\left(0, T ; H^{1}(\Omega)\right) .
\end{aligned}
$$


Here $\psi_{m}$ is the inverse of $\beta_{m}$, and so $\widetilde{w}_{m}=\psi_{m}\left(\widetilde{u}_{m}\right)$. We note, on recalling (3.19) again, that $\psi_{m}^{\prime}\left(\beta_{m}(s)\right)=\left[\beta_{m}^{\prime}(s)\right]^{-1} \in(0,1]$ for all $s \in \mathbb{R}$ and all $m \in \mathbb{N}$.

It follows from (3.7c) and Lemma 3.1 that $f \in[-M, 0]$ a.e. in $\Omega_{T}$, where $M:=\max \left\{r_{1}, r_{2}\right\}$. Choosing $\zeta=\left[\widetilde{u}_{m}-1\right]_{+} \chi_{[0, \widehat{t}]}$, for any $\widehat{t} \in[0, T]$, in (3.25) then yields that

$$
\frac{1}{2} \int_{0}^{\widehat{t}} \frac{d}{d t}\left(\int_{\Omega}\left[\widetilde{u}_{m}-1\right]_{+}^{2} \mathrm{~d} x\right) \mathrm{d} t+\int_{0}^{\widehat{t}} \int_{\Omega} \psi_{m}^{\prime}\left(\widetilde{u}_{m}\right)\left|\nabla\left[\widetilde{u}_{m}-1\right]_{+}\right|^{2} \mathrm{~d} x \mathrm{~d} t \leqslant 0 .
$$

Similarly, on choosing $\zeta=\left[\widetilde{u}_{m}+M t\right]_{-} \chi_{[0, \widehat{t}]}$ in (3.25), we deduce, as $\widetilde{u}_{m} \in C\left([0, T] ; L^{2}(\Omega)\right)$, that for all $t \in[0, T]$

$$
-M t \leqslant \widetilde{u}_{m}(x, t) \leqslant 1 \quad \text { for a.e. } x \in \Omega, \quad \text { for } m \in \mathbb{N} .
$$

Finally, on choosing $\zeta=\widetilde{u}_{m}$ in (3.25) and noting (3.27) yields for $m \in \mathbb{N}$ that

$$
\int_{0}^{T}\left|\nabla \psi_{m}\left(\widetilde{u}_{m}\right)\right|_{0}^{2} \mathrm{~d} t \leqslant C
$$

where the constant $C$ is independent of $m$.

Therefore, we have from (3.27) and (3.28) for any $m \in \mathbb{N}$ that $\widetilde{w}_{m} \in C\left([0, T] ; L^{2}(\Omega)\right) \cap$ $L^{2}\left(0, T ; H^{1}(\Omega)\right)$ with

$$
\left\|\widetilde{w}_{m}\right\|_{L^{\infty}\left(\Omega_{T}\right)}+\int_{0}^{T}\left|\nabla \widetilde{w}_{m}\right|_{0}^{2} \mathrm{~d} t \leqslant C
$$

It follows from subtracting (3.24) from (3.14) on choosing $\zeta(\cdot, t)=\int_{t}^{T}\left(\widetilde{w}-\widetilde{w}_{m}\right)(\cdot, s) \mathrm{d} s$ that for any $m \in \mathbb{N}$

$$
\int_{0}^{T}\left(\beta(\widetilde{w})-\beta_{m}\left(\widetilde{w}_{m}\right), \widetilde{w}-\widetilde{w}_{m}\right) \mathrm{d} t+\frac{1}{2} \int_{\Omega}\left|\nabla\left(\int_{0}^{T}\left[\widetilde{w}-\widetilde{w}_{m}\right] \mathrm{d} t\right)\right|^{2} \mathrm{~d} x=0
$$

Hence, we have from (3.30) that

$$
\begin{aligned}
\left\|\widetilde{w}-\widetilde{w}_{m}\right\|_{0, \Omega_{T}}^{2} & \leqslant \int_{0}^{T}\left(\beta_{m}(\widetilde{w})-\beta_{m}\left(\widetilde{w}_{m}\right), \widetilde{w}-\widetilde{w}_{m}\right) \mathrm{d} t \\
& \leqslant \int_{0}^{T}\left(\beta_{m}(\widetilde{w})-\beta(\widetilde{w}), \widetilde{w}-\widetilde{w}_{m}\right) \mathrm{d} t
\end{aligned}
$$

It follows from (3.31), (3.23) and (3.29) that

$$
\widetilde{w}_{m} \rightarrow \widetilde{w} \text { in } L^{2}\left(\Omega_{T}\right) \quad \text { and } \quad \widetilde{w}_{m} \rightarrow \widetilde{w} \text { in } L^{2}\left(0, T ; H^{1}(\Omega)\right) .
$$

Therefore, assumption [ $\mathrm{A}_{4}$ ] in [3] holds. Finally, the argument in [3] is a local one , and thus the weak formulation (2.1) in [3] for $\widetilde{w}$ is independent of initial and boundary conditions. This can be obtained by choosing $\left.\zeta=\phi \chi_{[} t_{a}, t_{b}\right]$ in (3.25) for any $\phi \in L^{2}\left(0, T ; H_{0}^{1}(\Omega)\right) \cap H^{1}\left(0, T ; L^{2}(\Omega)\right)$ and $0<t_{a}<t_{b} \leqslant T$, performing integration by parts in time, and passing to the limit $m \rightarrow \infty$ and noting (3.32).

Then from Theorem 1 in [3] we deduce that $\widetilde{w} \in C\left(\Omega_{T}\right)$, and so $\widetilde{u}=\beta(\widetilde{w}) \in C\left(\Omega_{T}\right), u=$ $1-\widetilde{u} \in C\left(\Omega_{T}\right)$ and the set $U$ is open. 
We now identify $F_{i} \in L^{2}\left(0, T ;\left[L^{2}(\Omega)\right]^{d}\right)$ appearing in $(3.2 \mathrm{~d})$, where we denote the $j^{\text {th }}$ component of $F_{i}$ by $F_{i, j}$. We recall from (3.11) that $F_{1}+F_{2}=-\nabla \psi(\widetilde{u})=-\frac{1}{2} \nabla[b(u)]^{2}$.

LEMMA 3.5 We have that

$$
\begin{aligned}
\frac{\partial u_{i}}{\partial x_{j}} \in L_{\mathrm{loc}}^{2}(u) \text { and } \quad F_{i}=\chi u b(u) \nabla u_{i} \quad \text { a.e. in } \Omega_{T}, \\
\quad i=1,2, \quad j=1, \ldots, d ;
\end{aligned}
$$

where $U$ is the open set $\left\{(x, t) \in \Omega_{T}: b(u)(x, t)>0\right\}$.

Proof. We begin by proving that $\frac{\partial}{\partial x_{j}}\left([b(u)]^{5} u_{i}\right) \in L^{2}\left(\Omega_{T}\right)$ for $i=1,2$ and $j=1, \ldots, d$. To begin, fix $\zeta \in C_{0}^{\infty}\left(\Omega_{T}\right)$; then Lemma 3.2, (3.11) and (3.2d) yield that

$$
\begin{aligned}
\int_{\Omega_{T}} & {[b(u)]^{5} u_{i} \frac{\partial \zeta}{\partial x_{j}} \mathrm{~d} x \mathrm{~d} t \leftarrow \int_{\Omega_{T}}\left[b\left(U_{h, \tau}^{-}\right)\right]^{5} U_{i ; h, \tau}^{-} \frac{\partial \zeta}{\partial x_{j}} \mathrm{~d} x \mathrm{~d} t } \\
& =-\int_{\Omega_{T}}\left[\frac{5}{2} \frac{\partial\left[b\left(U_{h, \tau}^{-}\right)\right]^{2}}{\partial x_{j}}\left[b\left(U_{h, \tau}^{-}\right)\right]^{3} U_{i ; h, \tau}^{-}+\left[b\left(U_{h, \tau}^{-}\right)\right]^{5} \frac{\partial U_{i ; h, \tau}^{-}}{\partial x_{j}}\right] \zeta \mathrm{d} x \mathrm{~d} t \\
& \rightarrow-\int_{\Omega_{T}}\left[\frac{5}{2} \frac{\partial[b(u)]^{2}}{\partial x_{j}}[b(u)]^{3} u_{i}+[b(u)]^{4} F_{i, j}\right] \zeta \mathrm{d} x \mathrm{~d} t
\end{aligned}
$$

for a subsequence of $\{h, \tau\} \rightarrow\{0,0\}$ satisfying (2.8). This implies that

$$
\frac{\partial\left([b(u)]^{5} u_{i}\right)}{\partial x_{j}}=\frac{5}{2} \frac{\partial[b(u)]^{2}}{\partial x_{j}}[b(u)]^{3} u_{i}+[b(u)]^{4} F_{i, j}, \quad i=1,2, \quad j=1, \ldots, d .
$$

We now use this relation in order to identify $F_{i}, i=1,2$, on the set $U$. To this purpose, let $\zeta \in C_{0}^{\infty}(u)$; then there exists a constant $\gamma>0$ such that $b(u) \geqslant \gamma$ on supp $\zeta$. Hence, we have that

$$
\begin{aligned}
& \int_{u} u_{i} \frac{\partial \zeta}{\partial x_{j}} \mathrm{~d} x \mathrm{~d} t=\int_{u}[b(u)]^{5} u_{i}[b(u)]^{-5} \frac{\partial \zeta}{\partial x_{j}} \mathrm{~d} x \mathrm{~d} t \\
& =-\int_{u}\left[\frac{\partial\left([b(u)]^{5} u_{i}\right)}{\partial x_{j}}[b(u)]^{-5}-\frac{5}{2}[b(u)]^{5} u_{i} \frac{\partial[b(u)]^{2}}{\partial x_{j}}[b(u)]^{-7}\right] \zeta \mathrm{d} x \mathrm{~d} t \\
& =-\int_{u}[b(u)]^{-1} F_{i, j} \zeta \mathrm{d} x \mathrm{~d} t
\end{aligned}
$$

that is, $F_{i}=b(u) \nabla u_{i}$ on $U, i=1,2$. As $F_{i} \in L^{2}\left(0, T ;\left[L^{2}(\Omega)\right]^{d}\right], i=1,2$, the first result in (3.33) follows.

It remains to identify $F_{i}$ on the complement of $U$. For any $\zeta \in C_{0}^{\infty}\left(\Omega_{T}\right)$, it follows, on noting 
(2.14a), that

$$
\begin{aligned}
& \left|\int_{\Omega_{T}} b\left(U_{h, \tau}^{-}\right) \frac{\partial U_{i ; h, \tau}^{-}}{\partial x_{j}}(1-\chi u) \zeta \mathrm{d} x \mathrm{~d} t\right| \\
& \leqslant\left(\int_{\Omega_{T}} b\left(U_{h, \tau}^{-}\right)\left|\nabla U_{i ; h, \tau}^{-}\right|^{2} \mathrm{~d} x \mathrm{~d} t\right)^{\frac{1}{2}}\left(\int_{\Omega_{T}} b\left(U_{h, \tau}^{-}\right)(1-\chi u) \zeta^{2} \mathrm{~d} x \mathrm{~d} t\right)^{\frac{1}{2}} \\
& \quad \leqslant C\left(\int_{\Omega_{T}}\left|b\left(U_{h, \tau}^{-}\right)-b(u)\right|(1-\chi u) \mathrm{d} x \mathrm{~d} t\right)^{\frac{1}{2}}
\end{aligned}
$$

since $b(u)(1-\chi u)=0$ a.e. in $\Omega_{T}$. On noting (3.2d) and (3.3a), we deduce that

$$
\int_{\Omega_{T}} F_{i, j}(1-\chi u) \zeta=0 \quad \forall \zeta \in C_{0}^{\infty}\left(\Omega_{T}\right), \quad i=1,2, \quad j=1, \ldots, d .
$$

Therefore $F_{i} \equiv 0$ a.e. on $\Omega_{T} \backslash U, i=1,2$. Hence, we have the second result in (3.33).

THEOREM 3.1 The pair $\left\{u_{1}, u_{2}\right\}$ is a weak solution of $(1.4 \mathrm{a})-(1.4 \mathrm{c})$ in the sense of Definition 1.1.

Proof. It follows from Lemmas 3.1, 3.4 and 3.5 that points (a)-d) hold in Definition 1.1. We have from (2.7) that for $i=1,2$ the function $U_{i ; h, \tau}$ satisfies $U_{i ; h, \tau}(\cdot, 0)=I^{h} u_{i}^{0}(\cdot)$ and

$$
\begin{array}{r}
\int_{0}^{T}\left[\left(\frac{\partial U_{i ; h, \tau}}{\partial t}, \chi\right)^{h}+\left(b\left(U_{h, \tau}^{-}\right) \nabla U_{i ; h, \tau}^{-}, \nabla \chi\right)\right] \mathrm{d} t=r_{i} \int_{0}^{T}\left(b\left(U_{h, \tau}^{-}\right) U_{i ; h, \tau}^{-}, \chi\right)^{h} \mathrm{~d} t \\
\forall \chi \in L^{1}\left(0, T ; S^{h}\right) .
\end{array}
$$

Applying integration by parts in time, and noting (2.3f) we deduce that for $i=1,2$ and for all $\chi \in H^{1}\left(0, T ; H^{1}(\Omega)\right)$

$$
\begin{aligned}
& \left|\int_{0}^{T}\left[\left(\frac{\partial U_{i ; h, \tau}}{\partial t}, \chi\right)-\left(\frac{\partial U_{i ; h, \tau}}{\partial t}, \chi\right)^{h}\right] \mathrm{d} t\right| \\
& \quad \leqslant C h \sup _{t \in(0, T)}\left\|U_{i ; h, \tau}(\cdot, t)\right\|_{0}\left(\int_{0}^{T}\left[\left\|\frac{\partial \chi}{\partial t}\right\|_{1}^{2}+\|\chi\|_{1}^{2}\right] \mathrm{d} t\right)^{\frac{1}{2}} .
\end{aligned}
$$

Let $\chi=I^{h} \zeta$ for any $\zeta \in C^{\infty}\left(\overline{\Omega_{T}}\right)$. Then passing to the limit $\{h, \tau\} \rightarrow\{0,0\}$ in (3.35) with $\chi=I^{h} \zeta$, for any $\zeta \in C^{\infty}\left(\overline{\Omega_{T}}\right)$, on noting (3.36), (2.3f), (2.3d), Lemmas 3.1 and 3.5, and (3.3a) yields that

$$
\int_{0}^{T}\left\langle\frac{\partial u_{i}}{\partial t}, \zeta\right\rangle_{H^{1}(\Omega)} \mathrm{d} t+\int_{u} b(u) \nabla u_{i} \cdot \nabla \zeta \mathrm{d} x \mathrm{~d} t=r_{i} \int_{\Omega_{T}} b(u) u_{i} \zeta \mathrm{d} x \mathrm{~d} t
$$

for all $\zeta \in C^{\infty}\left(\overline{\Omega_{T}}\right)$, with $u_{i}(\cdot, 0)=u_{i}^{0}(\cdot)$. Hence, the desired result (e) in Definition 1.1 follows on noting a density result for the test functions $\zeta$. Therefore, $\left\{u_{1}, u_{2}\right\}$ is a weak solution of (1.4a) $-(1.4 \mathrm{c})$ in the sense of Definition 1.1 


\section{The full model}

In this section we adapt the analysis in the previous two sections to the full model, $\left(\mathrm{P}_{z}\right),(1.1 \mathrm{a})-$ (1.1d), involving $z$, the $H^{+}$ion concentration.

We introduce the following finite element approximation of $\left(\mathrm{P}_{z}\right)$ : With $U_{i}^{0}=I^{h} u_{i}^{0}, i=1,2$, and $Z^{0}=I^{h} z^{0}$ for $n=1, \ldots, N$ find $\left\{U_{1}^{n}, U_{2}^{n}, Z^{n}\right\} \in S^{h} \times S^{h} \times S^{h}$ such that for $i=1,2$

$$
\begin{aligned}
\left(\frac{U_{i}^{n}-U_{i}^{n-1}}{\tau}, \chi\right)^{h}+\left(G_{i}\left(Z^{n-1}\right) U_{i}^{n}, \chi\right)^{h}+\left(b\left(U^{n-1}\right) \nabla U_{i}^{n-1}, \nabla \chi\right) & \\
& =r_{i}\left(b\left(U^{n-1}\right) U_{i}^{n-1}, \chi\right)^{h} \quad \forall \chi \in S^{h},
\end{aligned}
$$

and

$$
\begin{aligned}
&\left(\frac{Z^{n}-Z^{n-1}}{\tau}, \chi\right)^{h}+\alpha\left(\nabla Z^{n}, \nabla \chi\right)+\delta_{3}\left(Z^{n}, \chi\right)^{h}=\left(r_{3} U_{2}^{n}+\delta_{3}, \chi\right)^{h} \\
& \forall \chi \in S^{h}
\end{aligned}
$$

Once again, as $G_{i}(\cdot) \geqslant 0$, one can solve (4.1a) explicitly for $U_{i}^{n}, i=1,2$. As the given positive parameter $\alpha$ can be very large in the modelling of tumour growths, see [5] and the next section, we chose to make (4.1b) implicit in $Z^{n}$; as an explicit scheme would be impractical due to the severe constraint on the choice of time step $\tau$ for stability. The scheme (4.1b) requires a linear solve for $Z^{n}$ involving a sparse symmetric positive definite matrix. Hence, there exists a unique solution $\left\{U_{1}^{n}, U_{2}^{n}, Z^{n}\right\}$ to (4.1 a) and (4.1b). Of course (1.1a), (1.1b) and (4.1a), $i=1,2$, collapse to (1.4a), (1.4b) and (2.7), $i=1,2$, if $\delta_{1}=\delta_{2}=0$.

The bounds (2.9) of Lemma 2.1 remain true for (4.1a) and (4.1b) since the only changes to the proof, as stated, are that $m_{j} U_{i}^{n}\left(p_{j}\right)$ is replaced by $m_{j}\left(1+\tau G_{i}\left(Z^{n-1}\left(p_{j}\right)\right)\right) U_{i}^{n}\left(p_{j}\right)$ on the left-hand sides of (2.10) and (2.11), and $m_{j} U^{n}\left(p_{j}\right)$ is replaced by $m_{j}\left(U^{n}\left(p_{j}\right)+\right.$ $\left.\tau \sum_{i=1}^{2} G_{i}\left(Z^{n-1}\left(p_{j}\right)\right) U_{i}^{n}\left(p_{j}\right)\right)$ on the left-hand sides of (2.12) and (2.13). As $G_{i}(\cdot) \geqslant 0$, the desired results hold. In addition, on choosing $\chi=I^{h}\left[Z^{n}-\min \left\{z_{\min }^{0}, 1\right\}\right]_{-}$, where $z_{\min }^{0}=$ $\min _{x \in \bar{\Omega}} z^{0}(x) \geqslant 0$, in (4.1b) yields, on noting (1.3), (2.6) and (2.9), that, under assumption (2.8),

$$
Z^{n}(x) \geqslant \min \left\{z_{\min }^{0}, 1\right\} \quad \text { for all } x \in \bar{\Omega}, \quad n=0, \ldots, N \text {. }
$$

The bounds (2.14a) and (2.14b) of Lemma 2.2 remain true for (4.1a) and (4.1b). This is easily seen, as $G_{i}(\cdot) \in\left[0, \delta_{i}\right], i=1,2$, implies that (2.15) and (2.17) still hold. Similarly, on choosing $\chi=Z^{n}$ and $\chi=Q^{h} \zeta$ in (4.1b) yields, together with (2.3a), that

$$
\begin{array}{r}
\max _{n=0, \ldots, N}\left\|Z^{n}\right\|_{0}^{2}+\sum_{n=0}^{N} \tau\left\|Z^{n}\right\|_{1}^{2} \leqslant C, \\
\sum_{n=1}^{N}\left\|Z^{n}-Z^{n-1}\right\|_{0}^{2}+\sum_{n=1}^{N} \tau\left\|\frac{Z^{n}-Z^{n-1}}{\tau}\right\|_{\left(H^{1}(\Omega)\right)^{\prime}}^{2} \leqslant C .
\end{array}
$$

Clearly, the convergence results $(3.2 \mathrm{a})-(3.2 \mathrm{~d})$ of Lemma 3.1 remain true under the stated assumptions. In addition, it follows from (4.3a) and (4.3b), on adopting the notation (3.1a) and 
(3.1b) for $Z^{n}$ and noting (1.6), that for a subsequence of the sequence $\{h, \tau\}$ in Lemma 3.1

$$
\begin{aligned}
& Z_{h, \tau}, Z_{h, \tau}^{ \pm} \quad \rightarrow \quad z \quad \text { in } L^{2}\left(0, T ; H^{1}(\Omega)\right), \\
& \frac{\partial Z_{h, \tau}}{\partial t} \rightarrow \frac{\partial z}{\partial t} \quad \text { in } L^{2}\left(0, T ;\left(H^{1}(\Omega)\right)^{\prime}\right), \\
& Z_{h, \tau}, Z_{h, \tau}^{ \pm} \rightarrow z \quad \text { in } L^{2}\left(\Omega_{T}\right)
\end{aligned}
$$

where $z(x, t) \geqslant \min \left\{z_{\min }^{0}, 1\right\}$ for a.e. $(x, t) \in \Omega_{T}$.

The results (3.3a) and (3.3b) of Lemma 3.2 remain true, as (3.5) still holds since $G_{i}(\cdot) \in\left[0, \delta_{i}\right]$.

Similarly, on noting (4.4c) and that $G_{i} \in C\left(\mathbb{R} ;\left[0, \delta_{i}\right]\right), i=1,2$, the results of Lemmas 3.3 and 3.4 remain true with $f:=-b(u)\left(r_{1} u_{1}+r_{2} u_{2}\right)+G_{1}(z) u_{1}+G_{2}(z) u_{2}$.

Finally, the proof of Theorem 3.1 is easily modified, on noting (4.4a)-(4.4c) and that $G_{i} \in$ $C\left(\mathbb{R} ;\left[0, \delta_{i}\right]\right), i=1,2$, to show that $\left\{u_{1}, u_{2}, z\right\}$ is a weak solution of (1.1a)-(1.1d) in the sense of Definition 1.1 with (e) replaced by $z \in L^{2}\left(0, T ; H^{1}(\Omega)\right) \cap H^{1}\left(0, T ;\left(H^{1}(\Omega)\right)^{\prime}\right), z \geqslant \min \left\{z_{\min }^{0}, 1\right\}$ a.e. in $\Omega_{T}$,

$$
\begin{array}{r}
\int_{0}^{T}\left\langle\frac{\partial u_{i}}{\partial t}, \zeta\right\rangle_{H^{1}(\Omega)} \mathrm{d} t+\int_{u} b(u) \nabla u_{i} \cdot \nabla \zeta \mathrm{d} x \mathrm{~d} t+\int_{\Omega_{T}} G_{i}(z) u_{i} \zeta \mathrm{d} x \mathrm{~d} t \\
=r_{i} \int_{\Omega_{T}} b(u) u_{i} \zeta \mathrm{d} x \mathrm{~d} t \quad \forall \zeta \in L^{2}\left(0, T ; H^{1}(\Omega)\right) \quad i=1,2
\end{array}
$$

with $u_{i}(\cdot, 0)=u_{i}^{0}(\cdot)$; and

$$
\begin{array}{r}
\int_{0}^{T}\left\langle\frac{\partial z}{\partial t}, \zeta\right\rangle_{H^{1}(\Omega)} \mathrm{d} t+\alpha \int_{\Omega_{T}} \nabla z \cdot \nabla \zeta \mathrm{d} x \mathrm{~d} t=\int_{\Omega_{T}}\left[r_{3} u_{2}-\delta_{3}(z-1)\right] \zeta \mathrm{d} x \mathrm{~d} t \\
\forall \zeta \in L^{2}\left(0, T ; H^{1}(\Omega)\right)
\end{array}
$$

with $z(\cdot, 0)=z^{0}(\cdot)$.

\section{Numerical experiments}

All experiments were performed in MATLAB.

Firstly, in order to test our approximation, (4.1a) and (4.1b), of $\left(\mathrm{P}_{z}\right)$ we considered an appropriate extension of our scheme to the more general system, where (1.1a) and (1.1c) are replaced by

$$
\begin{aligned}
\frac{\partial u_{i}}{\partial t}-\nabla \cdot\left(b\left(u_{1}+u_{2}\right) \nabla u_{i}\right) & =r_{i} b\left(u_{1}+u_{2}\right) u_{i}-G_{i}(z) u_{i}+f_{i} & & \text { in } \Omega_{T}, \\
\frac{\partial z}{\partial t}-\alpha \nabla^{2} z & =r_{3} u_{2}-\delta_{3}(z-1)+f_{3} & & \text { in } \Omega_{T} .
\end{aligned}
$$

We chose $\Omega=(0,1)^{2}, T=0.1, r_{1}=r_{2}=r_{3}=\alpha=\delta_{3}=1$ and the functions $G_{i}$, (1.2a), with

$$
\delta_{1}=\delta_{2}=2, \quad z_{1}^{\mathrm{opt}}=1, \quad z_{2}^{\mathrm{opt}}=4, \quad z_{1}^{\text {width }}=0.1, \quad z_{2}^{\text {width }}=0.4
$$


TABLE 1

Errors and experimental orders of convergence (eocs) in $L^{\infty}\left(L^{2}\right)$ for test problem with known analytic solution.

\begin{tabular}{lllllll}
\hline$h$ & $E_{\infty, 0, u_{1}}$ & $e o c$ & $E_{\infty, 0, u_{2}}$ & $\rho o c$ & $E_{\infty, 0, z}$ & $e o c$ \\
\hline 0.14142 & 0.031701 & - & 0.032161 & - & $9.2161 \mathrm{e}-04$ & - \\
0.07071 & 0.007320 & 2.115 & 0.0074374 & 2.112 & $2.4341 \mathrm{e}-04$ & 1.921 \\
0.03536 & 0.001793 & 2.030 & 0.0018223 & 2.029 & $6.1779 \mathrm{e}-05$ & 1.979 \\
0.01768 & $4.4597 \mathrm{e}-04$ & 2.007 & $4.5327 \mathrm{e}-04$ & 2.007 & $1.5507 \mathrm{e}-05$ & 1.994 \\
0.01179 & $1.9802 \mathrm{e}-04$ & 2.004 & $2.0126 \mathrm{e}-04$ & 2.004 & $6.8972 \mathrm{e}-06$ & 2.000 \\
\hline
\end{tabular}

The functions

$$
\begin{aligned}
u_{1}(x, t) & =\sin (6 \pi t)\left[\cos \left(2 \pi x_{1}\right)\right]^{2}, \quad u_{2}(x, t)=\sin (6 \pi t)\left[\sin \left(2 \pi x_{1}\right)\right]^{2}, \\
z(x, t) & =e^{t} x_{2}^{2}\left(1-x_{2}\right)^{2}
\end{aligned}
$$

are non-negative and satisfy $u_{1}+u_{2} \leqslant 1$ over the considered time interval. In addition, they satisfy the homogeneous Neumann boundary conditions (1.1b), (1.1d) as well as the above system for suitably defined right-hand sides $f_{1}, f_{2}, f_{3}$. Note that $b\left(u_{1}(\cdot, t)+u_{2}(\cdot, t)\right) \equiv 0$ for $t=\frac{1}{12}$ so that the diffusion coefficient in the first two equations vanishes inside the considered time interval.

For a uniform mesh, obtained by dividing $\Omega$ into squares and then into triangles by introducing the NW-SE diagonal, of grid size $h$ we calculated the following errors, for convenience,

$$
\begin{aligned}
E_{\infty, 0, u_{i}}(h) & :=\max _{n=0, \ldots, N}\left\|U_{i}^{n}-u_{i}(\cdot, n \tau)\right\|_{0}, \\
E_{2,1, u_{i}}(h) & :=\left(\sum_{n=1}^{N} \tau\left\|\nabla\left(U_{i}^{n}-\left(I^{h} u_{i}\right)(\cdot, n \tau)\right)\right\|_{0}^{2}\right)^{\frac{1}{2}}
\end{aligned}
$$

for $i=1,2$ with analogous definitions for $E_{\infty, 0, z}(h)$ and $E_{2,1, z}(h)$. In practice, the error $E_{\infty, 0, \cdot}(h)$ was calculated using a numerical integration rule which samples at the centers of the triangles. The time step $\tau$ was chosen as $\tau=0.1 h^{2}$ so the stability bound (2.8) is satisfied. Tables 1 and 2 show the errors along with the experimental orders of convergence. We note that these errors converge at the optimal rate, which is possibly to be expected for this model degenerate problem as the underlying solution is smooth.

Secondly, we tested our approximation, (4.1a) and (4.1b), on data arising from the acid-mediated tumour invasion model. For the two-component system (P), (1.4a)-(1.4c), then any functions $u_{i} \geqslant$ $0, i=1,2$, with $u_{1}+u_{2}=1$ is a steady state. Whereas, constant steady states $\left\{u_{1}, u_{2}, z\right\}$ for the system $\left(\mathrm{P}_{z}\right),(1.1 \mathrm{a})-(1.1 \mathrm{~d})$, with $u_{i} \geqslant 0, i=1,2, u_{1}+u_{2} \leqslant 1$ and $z \geqslant 0$, satisfy

$$
G_{i}(z) u_{i}=r_{i}\left(1-\left(u_{1}+u_{2}\right)\right) u_{i}, \quad i=1,2, \quad \text { and } \quad \delta_{3}(z-1)=r_{3} u_{2} .
$$

Therefore if $\delta_{3}>0$ then one steady state is $\{0,0,1\}$, and another satisfies

$$
\left\{u_{1}, 0,1\right\}, \quad \text { where } \quad G_{1}(1)=r_{1}\left(1-u_{1}\right) .
$$


TABle 2

Errors and experimental orders of convergence (eocs) in $L^{2}\left(H^{1}\right)$ for test problem with known analytic solution.

\begin{tabular}{lllllll}
\hline$h$ & $E_{2,1, u_{1}}$ & $e o c$ & $E_{2,1, u_{2}}$ & $e o c$ & $E_{2,1, z}$ & $e o c$ \\
\hline 0.14142 & 0.231705 & - & 0.231705 & - & 0.005394 & - \\
0.07071 & 0.113071 & 1.035 & 0.113071 & 1.035 & 0.002545 & 1.084 \\
0.03536 & 0.056124 & 1.011 & 0.056124 & 1.011 & 0.001250 & 1.026 \\
0.01768 & 0.027986 & 1.004 & 0.027986 & 1.004 & $6.2124 \mathrm{e}-04$ & 1.009 \\
0.01179 & 0.018649 & 1.002 & 0.018649 & 1.002 & $4.1371 \mathrm{e}-04$ & 1.003 \\
\hline
\end{tabular}

Hence, if, in addition, $z_{1}^{\text {opt }}=1$ and $r_{1}>0$ then $\{1,0,1\}$ is a steady state. We chose the following data:

$$
\begin{aligned}
& \alpha=2.5 \times 10^{4}, \quad r_{1}=r_{2}=\alpha, \quad r_{3}=\delta_{3}=70 \alpha, \quad \delta_{1}=25 \alpha, \quad \delta_{2}=0.2 \alpha \\
& z_{1}^{\mathrm{opt}}=1, \quad z_{2}^{\mathrm{opt}}=2, \quad z_{1}^{\text {width }}=0.1, \quad z_{2}^{\text {width }}=0.4
\end{aligned}
$$

The majority of this data is derived from [4] after applying a rescaling, which is appropriate for the the present model; compare the definition of $\xi$ in (E) of [4] with that in (F) in [5]. Therefore the only constant steady states are $\{0,0,1\},\{1,0,1\}$ and $\{0,1,2\}$; that is, no cells, only normal cells and only tumour cells, respectively, with their associated optimal acidity level. The former state is unstable, whilst the other two are stable.

For $\lambda \in \mathbb{R}_{>0}$, we introduce the regularized Heaviside function

$$
H_{\lambda}(r):=0.5[\tanh (\lambda r)+1] .
$$

Then with $\Omega=(0,1)^{2}$, we considered initial data of the form

$$
\begin{aligned}
& u_{i}^{0}(x)=c_{i} H_{\lambda}\left(|x|-a_{i}\right)+b_{i}, \quad i=1,2, \\
& z^{0}(x)=c_{3} H_{\lambda}\left(|x|-a_{3}\right)+b_{3}
\end{aligned}
$$

where, for $i=1,2,3, a_{i} \in(0,1]$ and $b_{i}, c_{i} \in \mathbb{R}$. We chose

$$
\begin{aligned}
& \lambda=100, \quad a_{1}=a_{2}=a_{3}=0.1, \\
& b_{1}=0, \quad b_{2}=1, \quad b_{3}=2, \quad \text { and } \quad c_{1}=1, \quad c_{2}=c_{3}=-1 .
\end{aligned}
$$

This initial data connects smoothly the tumour cell steady state $\{0,1,2\}$ to the normal cell steady state $\{1,0,1\}$. We chose $T=0.0015$, and a uniform mesh as above with $h=\frac{\sqrt{2}}{50}$ and $\tau=$ $0.00125 h^{2}$. Contour plots from our approximation, (4.1a) and (4.1b), at the initial and final times are shown in Figure 1. Visually indistinguishable results are obtained for finer meshes. One sees the tumour edge is a travelling wave moving across the region preceded by a wave of higher acidity, which kills off normal cells leaving space to facilitate the tumour invasion. Of course this initial data is a steady state of the reduced approximation, (2.7), in the absence of $z$ as $u_{1}^{0}+u_{2}^{0}=1$. Finally, we note that the degeneracy of the diffusion coefficient $b\left(u_{1}+u_{2}\right)$ in (1.1a) does not appear to play such a crucial role in this tumour invasion model, as similar results to those that appear in Figure 1 can be achieved if $b\left(u_{1}+u_{2}\right)$ is replaced by unity. 

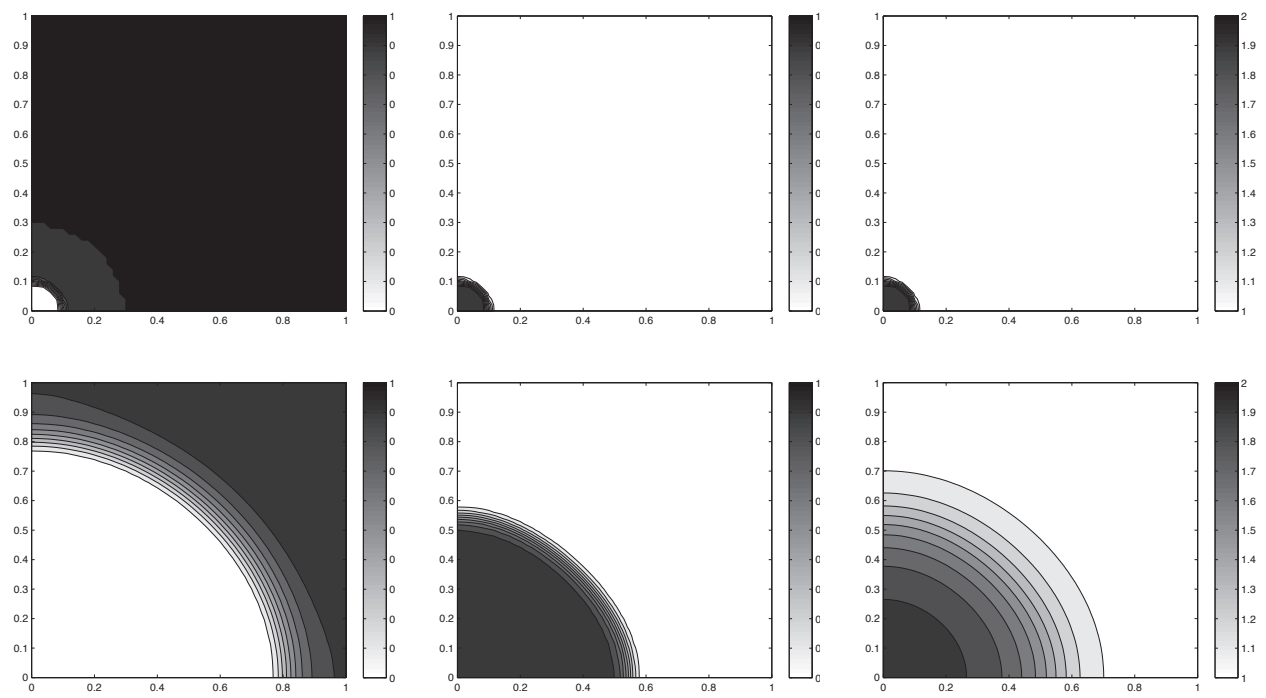

FIG. 1. Top: $U_{1}(\cdot, 0), U_{2}(\cdot, 0), Z(\cdot, 0)$, Bottom: $U_{1}(\cdot, T), U_{2}(\cdot, T), Z(\cdot, T)$.

\section{REFERENCES}

1. BARRETT, J.W. \& DeCKELNiCK, K. Existence, uniqueness and approximation of a doubly-degenerate nonlinear parabolic system modelling bacterial evolution. Math. Models Methods Appl. Sci. 17 (2007), 1095-1127. Zb11144.35026 MR2337432

2. Bertsch, M., Dal Passo, R. \& Mimura, M. A free boundary problem arising in a simplified tumour growth model of contact inhibition. Interfaces and Free Boundaries 12 (2010), 235-250. Zb11197. 35296 MR2652018

3. DiBenedetto, E. Continuity of weak solutions to a general porous medium equation. Indiana Univ. Math. J. 32 (1983), 83-118. Zbl0526. 35042 MR0684758

4. Gatenby, R.A. \& Gawlinski, E.T. A reaction-diffusion model for cancer invasion. Cancer Res. 56 (1996), 5745-5753.

5. Gatenby, R.A., Gawalinski, E.T., Gmitro, A.F., Kaylor, B. \& Gillies, R.J. Acid-mediated tumor invasion: A multidisciplinary study. Cancer Res. 66 (2006), 5216-5223.

6. KŘížEK, M., QUN, L. On diagonal dominance of stiffness matrices in 3D. East-West J. Numer. Math. 3 (1995), 59-69. Zbl0824.65112 MR1331484

7. Martin, K.M., Gaffney, E.A., Gatenby, R.A. \& Maini, P. Tumour-stromal interactions in acid mediated invasion: A mathematical model. J. Theor. Biology 267 (2010), 461-470.

8. Simon, J. Compact sets in the space $L^{p}(0, T ; B)$. Ann. Mat. Pura Appl. 146 (1987), 65-96. Zblo629. 46031 MR0916688

9. VÁzquez, J. The Porous Medium Equation, Oxford University Press. (2007). Zb11107.35003 MR2286292 